\title{
Capacity in Nonlinear Fiber Transmission Systems
}

A.D. Ellis, M.A. Sorokina, S. Sygletos, S.K. Turitsyn

Aston Institute of Photonic Technology, Aston University, Aston Triangle, James Watt Queensway, Birmingham, UK andrew.ellis@aston.ac.uk

\begin{abstract}
We review the nonlinear channel capacity of optical fiber communication systems using both linear and nonlinear amplifiers. We show that the capacity of a nonlinear transmission system employing linear optical amplifiers can be enhanced by over $300 \%$ by using all optical regeneration
\end{abstract}

OCIS codes: 070.4340 Nonlinear optical signal processing, 190.4380 Four-wave mixing.

\section{Introduction}

The capacity limit of a nonlinear fiber transmission system has been studied for many years [1-4], and is now the subject of tutorial and text book material [5-8]. The limits arise due to a tradeoff between amplified spontaneous emission noise and Kerr nonlinearity, either expressed as four wave mixing [9-10], cross phase modulation [4] or Gaussian noise [8] between different signal fields [1,2,4-9] and between signal and noise fields [3, 10-11]. With only a few exceptions $[2,3,8]$, all of these theories use the same basic assumptions, that different data bits interact in different fiber spans and that velocity matching is weak. This assumption essentially results in a complete randomization of the nonlinear distortion giving the same type of bi-Gaussian field distributions as observed for amplified spontaneous emission noise, resulting in a so called nonlinear signal to noise ratio $S N R_{N L}$. Under these approximations familiar expressions such as the Shannon limit and the bit error rate of various constellations [12] may be calculated by substituting the signal to noise ratio with the nonlinear signal to noise ratio.

Laboratory demonstrations are now able to match these theoretically predicted limits (for a comparative analysis see [13]), and in some specific cases even exceed these limits (for example [14-15]). However without exception, none of these cases represent a Memory Less Linear Channel with Additive White Gaussian Noise. This offers the intriguing prospect of operating a transmission system with a capacity beyond the nonlinear Shannon limit of $2 . \log _{2}\left(1+S N R_{N L}\right)$ through a controlled introduction/exploitation of either channel memory or beneficial nonlinear effects. In this paper, we consider the impact of of multi-wavelength phase sensitive regenerators [16-17] capable of regenerating higher order constellations [18-19] and their potential to offer significant increase in capacity.

\section{Nonlinear Shannon limit}

Assuming OFDM or Nyquist WDM super-channels occupying a bandwidth B, the nonlinear signal to noise ratio, taking into account ASE noise, inter channel nonlinearity, digital back propagation and parametric noise amplification (but neglecting PMD) is approximately given by;

$$
\begin{gathered}
S N R_{N L}=\frac{P_{S}}{N_{s} P_{\text {ase }}+N_{s}\left(\eta-\eta^{\prime}\right) P_{S}^{3}+\left(\sum_{n=1}^{N_{s}} n \eta-N_{S} \eta^{\prime}\right) P_{\text {ase }} P_{S}^{2}} \\
\eta=\frac{\omega_{0}^{2} n_{2}^{2}}{c^{2} \pi A_{\text {eff }}^{2} \alpha\left|\beta_{2}\right|} \cdot \ln \left(\frac{4 \pi^{2}\left|\beta_{2}\right| B^{2}}{2 \cdot \alpha}\right)
\end{gathered}
$$

where $P_{S}$ represents the signal power spectral density, $N_{S}$ the number of spans, $P_{\text {ase }}$ the amplified spontaneous emission power spectral density generated by each amplifier, $\eta$ a coefficient of nonlinearity depending on the WDM signal bandwidth B (and given by equation 2), $\eta$ ' represents the efficiency of digital back propagation, with a maximum value also given by equation 2 , but with the signal bandwidth $B$ replaced by the bandwidth subject to back propagation $B^{\prime}$. From left to right, the terms in the denominator of equation 1 represent amplifier spontaneous emission, inter channel nonlinearity and parametric noise amplification respectively. In equation 2 , the angular frequency at the center of the WDM band, the nonlinear refractive index, speed of light, effective area, fiber loss and dispersion coefficients have their usual symbols. From (1) it is possible to calculate the nonlinear Shannon capacity for a conventional system (neglecting parametric noise amplification for simplicity), a regenerated system (replacing the total number of spans $N_{S}$ with the number of spans between each of $N_{R}$ regenerators $\left({ }_{N} S /\left(1+N_{R}\right) 7\right)$ and a system with ideal digital back propagation $\left(\eta^{\prime}=\eta\right)$. For these cases, the maximum nonlinear signal to noise ratio is given by;

$$
S N R_{\text {Conv }}=\frac{1}{N_{S}} \sqrt[3]{\frac{4}{9 \eta P_{\text {ase }}^{2}}} \quad S N R_{\text {Re gen }}=\frac{1}{\left\lceil N_{S} /\left(1+N_{R}\right)\right\rceil \sqrt[3]{\frac{4}{9 \eta P_{\text {ase }}^{2}}}} \quad S N R_{D B P}=\frac{1}{N_{S}-1} \sqrt{\frac{1}{2 \eta P_{\text {ase }} N_{S}}}
$$


Note that here we neglect error propagation between regenerator spans [20]. From equation 3 we can immediately observe that ideal optical regeneration may improve the maximum signal to noise ratio directly in proportion to the number of regenerators used (up to a maximum improvement equal to the number of spans), such that $S N R_{\text {Regen }} \approx N_{R} \cdot S N R_{\text {Conv }}$. Similarly we would expect the capacity increase to asymptotically approach $\Delta C=B \cdot \log _{2}\left(1+N_{R}\right)$ In the case of digital back propagation, subject to the limits imposed by PMD, the maximum improvement in signal to noise ratio and increase in capacity is given by

$$
S N R_{D B P}=\sqrt{\left(\frac{3}{2}\right)^{3} \frac{N_{S}}{N_{S}-1} S N R_{\text {Conv }}^{3}} \quad, \quad \frac{\Delta C}{B}=0.877+\log _{2}\left(\frac{N_{S}}{N_{S}-1}\right)+\frac{1}{2} \frac{C}{B}
$$

\section{Nonlinear amplifiers}

In order to calculate the capacity of a given communication channel, it is necessary to calculate the conditional probability of receiving a particular point in the constellation diagram [4]. For an ideal cascade of phase regenerators (constant amplitude signals) the conditional probabilities for m-QAM with rectangular decision boundaries may be readily calculated to be

$$
P\left(x_{k} \mid x_{j}\right)=\left.\underline{\underline{W}}^{N_{R}}\right|_{k j} \quad W_{k j}=\frac{1}{2}\left(e f c\left(\sqrt{\frac{P_{S}}{2\left\lceil N_{S} /\left(1+N_{R}\right)\right\rceil P_{a s e}}} \frac{x_{k}+x_{k+1}-2 x_{j}}{2 \sqrt{\left\langle x_{k}^{2}\right\rangle}}\right)-e f c\left(\sqrt{\frac{P_{S}}{2\left\lceil N_{S} /\left(1+N_{R}\right)\right\rceil P_{a s e}}} \frac{x_{k}+x_{k-1}-2 x_{j}}{2 \sqrt{\left\langle x_{k}^{2}\right\rangle}}\right)\right)(5)
$$

where $\underline{\underline{W}}$ represents the transmission matrix of a single regenerator span, with matrix elements $W_{k j}, \digamma .7$ represents the rounding (up) operation, $x_{k}$ represents one coordinate of the field value laying within the decision boundary for the $k^{\text {th }}$ constellation point where the coordinate axes are aligned along the natural axes of the constellation and the magnitude of the constellation points increase in value with $k$. A similar expression exists for the $\mathrm{y}-$ coordinate. It may be shown that when the error probability is low (high signal to noise ratio within each regenerator span), the increase in capacity approaches

$$
\frac{\Delta C}{B}=\log _{2}\left(\frac{\pi \cdot e \cdot\left(N_{R}+1\right)}{4 \Delta}\right)+\frac{\left(N_{R}+1\right) \cdot e^{-\Delta^{2}}}{\Delta \sqrt{\pi}} \log _{2}\left(\frac{\left(N_{R}+1\right) \cdot e^{-\Delta^{2}}}{\Delta \sqrt{\pi}}\right) \quad \text { with } \quad \Delta^{2}=2\left(1+10 N_{R}^{-1}\right) \Omega\left(\frac{e^{2}\left(N_{R}+1\right)^{2}}{8 \cdot \pi\left(1+10 /\left(1+N_{R}\right)\right)}\right)(6)
$$

where $\Omega$ (.) represents the Lambert function. The first term of equation 6 represents the loss of capacity associated with the use of a discrete constellation whilst the second term represents the entropy change associate with the error propagation through a cascade of regenerators. For practical regenerators with continuous (not step-wise) transfer functions [eg 18], it may be necessary to calculate the transmission matrices $W_{k j}$ numerically.

\section{Typical performance}
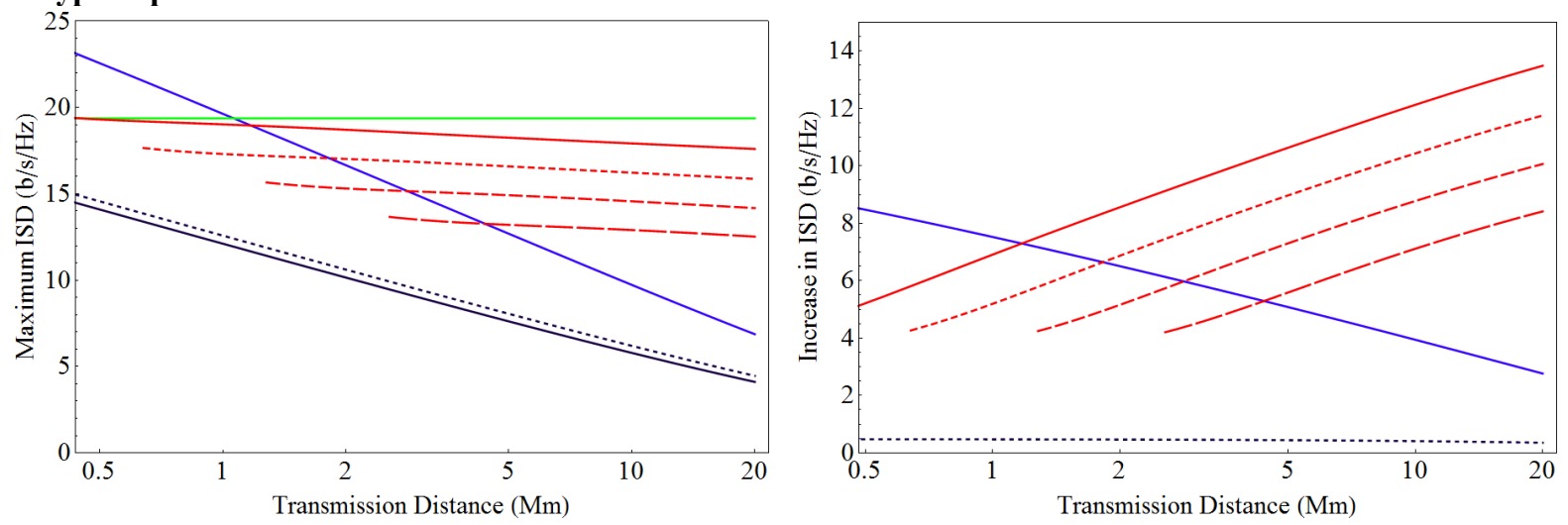

Figure 1; Left; Maximum capacity of a $5 \mathrm{THz}$ polarization multiplexed systems as a function of reach assuming $80 \mathrm{~km}$ amplifier spacing, $5 \mathrm{~dB}$ noise figures, using fibre with dispersion loss and nonlinear coefficients of $16 \mathrm{ps} / \mathrm{nm} / \mathrm{km}, 0.19 \mathrm{~dB} / \mathrm{km}$ and $1.4 / \mathrm{W} / \mathrm{km}$ respectively. Solid lines join discrete points calculated every $80 \mathrm{~km}$. Dark blue - conventional system with (dashed) and without (solid) digital back propagation covering a $250 \mathrm{GHz}$ bandwidth. Light blue - conventional system utilizing nonlinear compensation over the full system bandwidth. Green - using ideal optical regeneration without error propagation. Red-optical regeneration including error propagation. The optically regenerated systems are shown with one regenerator every 1 (solid), 2, 4 and 8 (long dashed) spans. Right; same data plotted as the increase in achievable capacity with respect to the conventional system.

Figure 1 illustrates typical performance characteristics for the conventional system with and without digital back propagation, and for optical regenerators showing calculations assuming no error accumulation between 
regenerators and calculations taking into account the full conditional probability distribution for a cascade of regenerators. Digital back-propagation of the channel of interest, or even over an optical bandwidth of $250 \mathrm{GHz}$ for an optical super channel with phase coherent receivers (shown as the dark blue dashed line) only offers a few $\mathrm{dB}$ improvement in SNR (or transmission reach), and whilst this is useful for extending the capacity of mesh networks [21] the benefit is restricted. Nonlinearity compensation over the full WDM bandwidth on the other hand offers a substantial increase in capacity [10] (shown as the light blue line) and may be implemented using optical phase conjugation [22]. This enables an approximately 50\% increase in capacity for all length scales. As indicated in equation 3, ideal optical regeneration restores the signal to noise ratio, essentially shortening the transmission span over which linear and nonlinear noise accumulate. This basic performance, neglecting error propagation is shown in green in figure 1 (solid green line). The exact calculation for a cascade of optical regenerators is shown by the series of red lines, ranging from one regenerator per span (solid red line) to one regenerator for eight spans (long dashed red line). Despite a slight deviation from the ideal regenerator, all optical regeneration offers significant capacity increases when compared to a conventional system, and presents the highest capacity solution over single mode fiber for transoceanic distances.

\section{Conclusions}

In this paper we have discussed the capacity limits of conventional WDM systems and compared them to the limits obtained using either nonlinearity compensation or all-optical regeneration. We find that both full band nonlinearity compensation and all-optical regeneration both offer significant capacity gains, despite error accumulation in the case of the all optical regeneration solutions. In particular we find that all optical regeneration offers the highest overall capacity for transoceanic systems and offers up to $75 \%$ increase in capacity for transcontinental systems.

\section{Acknowledgements}

The work has been supported by the EPSRC project UNLOC (Unlocking the capacity of optical communications), the Marie Currie - IEF project ARTISTE (IEF 330697) and The Royal Society (WM120035-TEST).

\section{References}

[1] A. Splett, C. Kurtzke, and K. Petermann, "Ultimate transmission capacity of amplified optical fiber communication systems taking into account fiber nonlinearities," ECOC'93, paper MoC2.4 (1993).

[2] J. Tang, "The Shannon channel capacity of dispersion-free nonlinear optical fiber transmission", JLT, 19, pp1104 (2000).

[3] K.S. Turitsyn, et al., "Information capacity of optical fiber channels with zero average dispersion", PRL, 91, pp203901 (2003).

[4] P.P. Mitra, J.B. Stark, "Nonlinear limits to the information capacity of optical fibre communications", Nature, 411, pp1027, (2001).

[5] A.D. Ellis, "Modulation Formats Which Approach the Shannon Limit", OFC 2009, paper OMM4, (2009).

[6] R.-J. Essiambre, et al., "Capacity limit of optical fiber networks", JLT, 28, 4, pp662, (2010).

[7] A.D. Ellis, J. Zhao, "Channel Capacity of Nonlinear Transmission Systems", in Impact of Nonlinearities on Fiber Optic Communications, ed. S. Kumar, Springer, New York (2011).

[8] P. Poggiolini, "Modeling of Non-Linear Propagation in Uncompensated Coherent Systems", OFC 2013, paper OTh3G.1 (2013).

[9] X. Chen and W. Shieh, "Closed-form expressions for nonlinear transmission performance of densely spaced coherent optical OFDM systems", Optics Express, 18, pp19039, (2010).

[10] D. Rafique and A. D. Ellis, "Impact of signal-ASE four-wave mixing on the effectiveness of digital back-propagation in $112 \mathrm{~Gb} / \mathrm{s}$ PM-QPSK systems", Optics Express, 19, pp3449, (2011).

[11] G. Gao, et al., "Influence of PMD on fiber nonlinearity compensation using digital back propagation", Optics Express, 20, pp14406, (2012).

[12] J.G. Proakis, Digital Communications (4th Edition), New York, McGraw-Hill (2000).

[13] A.D. Ellis, "Current trends in Optical Communications", Australian Conference on Optical Fiber Technology, Paper 10G.1 (2012).

[14] M. Nakazawa et al., "10 Gbit/s soliton data transmission over one million kilometres", Electronics Letters, 27, pp1270 (1991).

[15] Y. Cai et al., "Ultra long haul WDM transmission with high spectral efficiency", IEICE Transactions on Communications, E94-B, pp392,

(2011).

[16] S. Sygletos, et al., “A Practical Phase Sensitive Amplification Scheme for Two Channel Phase Regeneration”, Optics Express, 19, pp.B938 (2011).

[17] A.D. Ellis, and S. Sygletos, "Phase sensitive regeneration based on SOAs", OFC 2013, paper OW4C1, (2013)

[18] J. Kakande et al., "Multilevel quantization of optical phase in a novel coherent parametric mixer architecture", Nature Photonics, 5, pp748, (2011).

[19] T. Roethlingshoefer, et al., "Multilevel Amplitude and Phase Regeneration in a Nonlinear Amplifying Loop Mirror with a Phase-Sensitive Amplifier", ECOC12, paper Tu.1.A.3, (2012).

[20] A.D. Ellis, and N.J. Doran, “Are few mode fibres a solution to the capacity crunch?”, ICTON 2013, paper Tu.C2.1, (2013).

[21] D. Rafique, A.D. Ellis, "Nonlinear and ROADM induced penalties in 28 Gbaud dynamic optical mesh networks employing electronic signal processing”, Optics Express, 19, pp16739, (2011).

[22] M.D. Pelusi, "WDM Signal All-Optical Precompensation of Kerr Nonlinearity in Dispersion-Managed Fibers”, PTL, 25, pp71, (2013). 